\title{
Multidisziplinäre Untersuchungen zur nachhaltigen Stickstoffdüngung unter Berücksichtigung der Möglichkeiten der satellitengestützten Präzisionslandwirtschaft
}

\section{Multidisciplinary studies on sustainable nitrogen fertilisation considering the potential of satellite-based precision agriculture}

\author{
Laura Essl $^{1 *}$, Clement Atzberger ${ }^{1}$, Taru Sandén $^{2}$, Heide Spiegel$^{2}$, Julia Blasch ${ }^{3}$, Francesco Vuolo $^{1 *}$
}

\footnotetext{
${ }^{1}$ Institut für Geomatik, Universität für Bodenkultur Wien (BOKU), Peter-Jordan-Straße 82, 1190 Wien, Österreich

${ }^{2}$ Abteilung für Bodengesundheit und Pflanzenernährung, Österreichische Agentur für Gesundheit und Ernährungssicherheit (AGES), Spargelfeldstraße 191, 1220 Wien, Österreich

${ }^{3}$ Institute for Environmental Studies (IVM), Vrije Universiteit Amsterdam, De Boelelaan 1111, 1081 HV Amsterdam, Niederlande

* Korrespondierende Autoren: laura.essl@boku.ac.at und francesco.vuolo@boku.ac.at
}

Einreichung: 10. Dezember 2020, überarbeitete Einreichung: 1. Juni 2021, Annahme: 6. Juni 2021

\section{Zusammenfassung}

Im Forschungsprojekt FATIMA (FArming Tools for external nutrient Inputs and water MAnagement) wurde ein integrierter Ansatz für die Optimierung des Stickstoffmanagements in der Landwirtschaft gewählt. Neben der technischen Komponente, die die Verarbeitung von Sentinel-2-Satellitendaten umfasste, wurden auch die sozio-ökonomischen Aspekte eines verbesserten Nährstoffmanagements untersucht. Die Ergebnisse zeigen, dass LandwirtInnen vor allem an zeitnahen Applikationskarten zur teilflächenspezifischen Stickstoffausbringung interessiert sind. Im Zuge des Projekts wurde nicht nur ein Feldversuch für die Validierung der Satellitendaten als Grundlage einer ökonomischen Analyse angelegt, sondern auch eine funktionierende Online-Anwendung für teilflächenspezifische Stickstoffdüngung, basierend auf aktuellen und historischen Satellitenbildern, entwickelt und getestet.

Schlagwörter: Fernerkundung, Stickstoffdüngung, Feldversuche, Winterweizen, Choice Experiment, Applikationskarten

\section{Summary}

In the H2020 project FATIMA (FArming Tools for external nutrient Inputs and water MAnagement) an integrated approach for the optimisation of nitrogen management in agriculture was applied. In addition to the technical component, which included the processing of Sentinel-2 satellite data, the socio-economic and agronomic aspects of improved nutrient management were investigated. The results show that farmers are mainly interested in timely application maps for variable-rate nitrogen application. In the course of the project not only a field trial for the validation of satellite data as a basis for an economic analysis was established, but also an online application for site-specific fertilisation based on current and historical satellite images was developed and tested.

Keywords: remote sensing, nitrogen fertilisation, field experiments, winter wheat, choice experiment, application maps 


\section{Einleitung}

Weltweit tragen Stickstoffauswaschungen aus der Landwirtschaft zu erhöhtem Nitratgehalt im Grundwasser bei (Cameron et al., 2013; Rostenstock et al., 2014; Hansen et al., 2017). Die erhöhten Nitratwerte führen zu ökonomischen und ökologischen Problemen. Eine Nutzung von nitratbelastetem Grundwasser als Trinkwasser hat negative Auswirkungen auf die Gesundheit durch vermehrtes Auftreten von Methämoglobinämie bei Kindern und das erhöhte Risiko für das Auftreten bestimmter Krebserkrankungen (Ward et al., 2018). Die Lebensqualität von Menschen in belasteten Gebieten ist zudem eingeschränkt. Sie greifen zumeist auf teures, abgefülltes Wasser zurück oder reinigen das Hausbrunnenwasser in eigenen Aufbereitungsanlagen (Lewandowski et al., 2008; Juntakut et al., 2020). Auch die zentrale Aufbereitung von nitratbelastetem Grundwasser, um die gesetzlichen Auflagen zu erfüllen, ist kostspielig. Eine deutsche Studie ergab Gesamtkosten von 0,55-0,76€ $\mathrm{m}^{-3}$ behandeltem Wasser (Umweltbundesamt, 2017). Überschüssige Stickstoffderivate in Wasser und Luft tragen des Weiteren zu erhöhten Treibhausgasemissionen (Galloway et al., 2003; Snyder et al., 2009) und der Eutrophierung von Gewässern (Withers et al., 2014) bei.

Die Europäische Union legt in der Nitratrichtlinie 91/676/ EWG einen Grenzwert für das Grundwasser von $50 \mathrm{mg} \mathrm{l}^{-1}$ Nitrat Grundwasser fest. In Österreich wurde als Umsetzungsmaßnahme die Nitrat-Aktionsprogramm-Verordnung (BGBl. II Nr. 385/2017) beschlossen. Darin werden die maximalen Stickstoffmengen, die im Laufe eines Jahres ausgebracht werden dürfen, sowie die Aufzeichnungspflicht über Lagerung und Ausbringungsmengen abhängig von (Vor-)Kultur und Ertragslage festgelegt. In der Qualitätszielverordnung Chemie Grundwasser (BMLFUW, 2010) wird ein Wert von $45 \mathrm{mg} \mathrm{l}^{-1}$ zur Erreichung eines guten chemischen Zustands des Grundwassers festgelegt. Empfohlene Maßnahmen, die der Reduktion des Nitratgehalts dienen sollen, sind vermehrte Begrünung von Ackerflächen, Fruchtfolgeauflagen, verbesserte Düngeplanung und -ausbringung und Erstellung von Nährstoffbilanzen (BMLFUW, 2010).

Die aufgrund der Richtlinien für die sachgerechte Düngung (BMLFUW, 2017) empfohlene N-Düngermenge beläuft sich auf $110-130 \mathrm{~kg} \mathrm{ha}^{-1}$ Stickstoff $(\mathrm{N})$ bei mittlerer Ertragserwartung. Um den N-Bedarf genau zu bestimmen, empfiehlt die Richtlinie die Standorteigenschaften wie Bodenund Wasserverhältnisse, das N-Mineralisierungspotenzial
$\left(\mathrm{N}_{\text {min }}\right)$, die tatsächliche Ertragserwartung und die Vorfrucht zu berücksichtigen. Ein einfach zu bedienendes OnlineTool (AGES Agrarcommander) wird auf der Website der AGES (https://www.ages.at/service/service-landwirtschaft/ agrar-online-tools) bereitgestellt. Eine Stickstoffdüngungsempfehlung ist auch auf der Basis von Frühjahrs-NminAnalysen möglich.

Neben verstärkter Bewusstseinsbildung und finanziellen Anreizen für ein verbessertes Stickstoffmanagement im Zuge der Gemeinsamen Agrarpolitik der Europäischen Union ermöglichen es auch neue Technologien der einzughaltenden Präzisionslandwirtschaft das Nährstoffmanagement effizienter zu gestalten. Diese Technologien basieren zumeist auf bodennah oder -fern angebrachten optischen Sensoren. Die Erfassung des Vegetationsstatus mittels optischer Reflexion findet sowohl direkten Einsatz auf Landmaschinen, auf denen Sensoren pflanzenbezogene Indizes für den Stickstoffstatus messen und eine Stickstoffausbringungsrate empfehlen (Groher et al., 2020), als auch in der Satellitentechnologie. Im zweiten Fall befinden sich die Sensoren auf den Satelliten und messen mit einer festgelegten zeitlichen und räumlichen Auflösung den Wachstumsstatus der Pflanzen (Heege, 2013). Daraus können Zonen mit hohem und niedrigem Wachstum abgeleitet und darauf aufbauend die Düngerstrategie angepasst werden.

Das H2020-Forschungsprojekt FATIMA (FArming Tools for external Inputs and water Management) hatte zum Ziel, die durch Sentinel-2 verfügbaren Satellitendaten für ein verbessertes Stickstoffdüngemanagement zu verwenden. Bisher gibt es nur wenige Ansätze, die diese Daten der European Space Agency (ESA) in leicht anzuwendende Entscheidungshilfen für Landwirte umwandeln.

\section{Problemstellung und Projektgebiet}

Bei der Frage nach dem optimalen Stickstoffdüngereinsatz sind verschiedene Einflussfaktoren und teilweise konkurrierende Zielsetzungen zu berücksichtigen: wie viel Stickstoff bringt unter den gegebenen Standortbedingungen den maximalen Ertrag für eine bestimmte Kultur unter Berücksichtigung der Umwelterfordernisse und welche Anwendungsstrategie ist wirtschaftlich und ökologisch sinnvoll? An diesem Punkt setzte das EU-Forschungsprojekt FATIMA an. Einerseits wurde die Entwicklung von neuen Technologien basierend auf Fernerkundungsdaten der Sentinel-2-Satelliten vorangetrieben, andererseits wurden auch die sozio-ökonomischen und pflanzenbaulichen 
Komponenten der einzughaltenden „Landwirtschaft 4.0“ beleuchtet. Bisher wurden in Österreich weder Studien zur Akzeptanz von technologiegestütztem Stickstoffmanagement veröffentlicht noch die Nutzung von Satellitendaten zur Erstellung von Applikationskarten untersucht. Diese Studie verbindet sozio-ökonomische, pflanzenbauliche und die technischen Komponenten mit dem Ziel, in Zukunft akzeptierte, funktionsfähige und validierte Anwendungen für österreichische LandwirtInnen bereitzustellen.

Forscher der Universität für Bodenkultur (BOKU) und der österreichischen Agentur für Gesundheit und Ernährungssicherheit (AGES) untersuchten dabei insbesondere wie das Stickstoffmanagement mithilfe von Satellitendaten verbessert werden kann und ob LandwirtInnen in Österreich bereit für diesen Wandel sind.

Als Projektgebiet wurde das Marchfeld in Niederösterreich östlich von Wien gewählt. Das Marchfeld ist mit 60.000 ha landwirtschaftliche genutzte Fläche (Vuolo et al., 2018) eines der wichtigsten landwirtschaftlichen Produktionsgebiete Österreichs, wobei der Schwerpunkt auf Getreideund Gemüseanbau (Neugebauer und Vuolo, 2014) liegt. Die Ergebnisse des aktuellen Grundwasser-Monitorings des österreichischen Umweltbundesamtes zeigen, dass gerade in den intensiv landwirtschaftlich genutzten Regionen Ostösterreichs, in denen auch das Marchfeld liegt, die Nitratwerte über dem für Trinkwasser festgesetzten Wert von $50 \mathrm{mg} \mathrm{l}^{-1}$ liegen. Das Gebiet wurde daher als voraussichtliches Maßnahmengebiet (50\% der Messstellen überschreiten den Grenzwert für Nitrat) ausgew.iesen (Umweltbundesamt, 2016). Der niedrige Jahresniederschlag von $420 \mathrm{~mm}$ verstärkt das Problem, da dadurch das Sickerwasser eine höhere Nitratkonzentration aufweist (Wick et al., 2012). Auch die langsame Grundwasserneubildung im
Marchfeld (Nachtnebel et al., 2001) verschärft das Problem der Nitratbelastung, da Maßnahmen lange brauchen, um Wirkung zu zeigen.

\section{Materialien und Methoden}

Als erste Komponente in dem dreistufigen Prozess (Abbildung 1) wurde die Aufgeschlossenheit und das bestehende Wissen über satelliten- oder sensorunterstützte Düngung niederösterreichischer LandwirtInnen abgefragt. Parallel dazu wurden Versuchsflächen in der Region Marchfeld angelegt. Der Feldversuch diente der Validierung der Satellitendaten, gab aber auch Informationen über die pflanzenbauliche Komponente variabler Düngermengen. In einem letzten Schritt wurde eine Anwendung entwickelt, um die teilflächenspezifische Stickstoffdüngung auf zwei Flächen zu testen, die gemäß der landwirtschaftlichen Praxis bewirtschaftet wurden, um damit den Schritt von der Theorie hin zur Praxis zu bestreiten. Die Ergebnisse bieten ein umfassendes, multidisziplinäres Bild der Herausforderungen, aber auch Möglichkeiten von neuen Technologien im Bereich des N-Düngemanagements. Die drei methodischen Komponenten werden in den folgenden Kapiteln genauer geschildert.

\subsection{Sozio-ökonomische Komponente}

Um herauszufinden, ob eine Bereitschaft vorhanden ist, neue, teilflächenbasierte Lösungen im Bereich Stickstoffmanagement anzuwenden, startete die Freie Universität Amsterdam (IVM) in Kooperation mit der BOKU eine Umfrage unter niederösterreichischen LandwirtInnen. Die Umfrage wurde in persönlichen Interviews von

\section{Sozio-ökonomische Komponente}

Sind die LandwirtInnen

bereit für neue

Technologien im Bereich

N-Management?

\begin{tabular}{|l} 
Pflanzenbauliche Kom \\
$\begin{array}{l}\text { Wieviel Stickstoff ist die } \\
\text { optimale Menge? }\end{array}$
\end{tabular}

Wie kann der Stickstoff bestmöglich ausgebracht werden?

Optimiertes NManagement

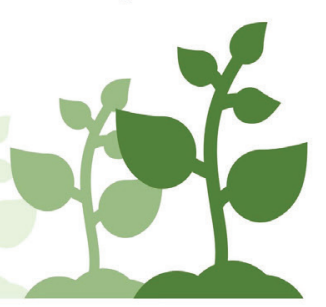

Abbildung 1. Der Weg zu einem verbesserten Stickstoffmanagement im Projekt FATIMA unter Berücksichtigung sozio-ökonomischer, pflanzenbaulicher und technischer Komponenten.

Figure 1. The approach to an improved nitrogen management in the FATIMA project taking into account socio-economic, agronomic and technical components. 
Studierenden der BOKU im Zeitraum November 2016 bis Februar 2017 mit Unterstützung der Landwirtschaftskammer Niederösterreich (NÖ) durchgeführt. Der Fragebogen bestand aus einem allgemeinen Teil mit Fragen zum Betrieb und einem Diskreten Entscheidungsexperiment (Choice Experiment), in dem die LandwirtInnen aus verschiedenen innovativen Optionen des Düngemanagements wählen konnten.

Den LandwirtInnen wurden drei Kategorien von Technologien für teilflächenspezifische Stickstoffausbringung vorgestellt:

- Voll automatisierte Technologien: automatisch gesteuerte variable Ausbringung des Stickstoffs auf Basis von Spektralmessungen durch den Sensor während der Fahrt über den Schlag.

- Teilweise automatisierte Technologien: mittels Satellitenaufnahmen erstellte Applikationskarten mit ausgewiesenen Zonen zur direkten Verwendung am Bordcomputer.

- Nicht automatisierte Technologien: mittels Satellitenaufnahmen erstellte Applikationskarten mit ausgewiesenen Zonen, ohne Möglichkeit des Transfers auf den Bordcomputer.

Den TeilnehmerInnen wurden die drei Optionen unter Berücksichtigung verschiedener Eigenschaften der einzelnen Technologien vorgestellt. Die drei vorgestellten Technologiegruppen wiesen beispielsweise unterschiedliche Kosten, N-Düngeeinsparungspotenziale, Ertragssteigerungspotenziale, Beratungsangebote und Grundwasserverbesserungspotenziale auf. Den LandwirtInnen wurden sogenannte choice cards präsentiert, auf denen sie die für ihren Betrieb am besten geeignete Lösung auswählen konnten. Auch die Wahl keines der drei Systeme war möglich. Aus den Antworten konnte die Investitionsbereitschaft, aber auch die Wichtigkeit der einzelnen Kriterien abgeleitet werden. Eine detaillierte Beschreibung der Methode findet sich bei Blasch et al. (2020).

An der Studie nahmen 242 niederösterreichische LandwirtInnen teil. Die befragten LandwirtInnen stammten aus den Regionen Marchfeld, Weinviertel, Wiener Becken und Tullnerfeld. Sie waren zumeist EigentümerInnen (82 \%) von Familienbetrieben (95 \%). Die Betriebsgrößen bewegten sich etwas über dem niederösterreichischen Durchschnitt von 42 ha (Grüner Bericht, 2016).

Zwei Drittel der befragten LandwirtInnen bewirtschafteten mehr als 50 ha, $26 \%$ sogar mehr als 100 ha.
Die LandwirtInnen waren zudem meist TeilnehmerInnen der Arbeitskreise der Landwirtschaftskammer Niederösterreich. Die Untersuchung der Charakteristika der UmfrageteilnehmerInnen ergibt einen im Vergleich zum Durchschnittsbetrieb (NÖ Agrarstrukturerhebung 2016) jüngeren, mit mehr landwirtschaftlich genutzter Fläche ausgestatteten und an Neuigkeiten im Agrarsektor interessierten männlichen Betriebsführer. Tabelle 1 gibt einen Überblick über die Betriebskennzahlen und Eigenschaften der LandwirtInnen.

Tabelle 1. Charakteristika und Betriebskennzahlen der teilnehmenden LandwirtInnen $(\mathrm{N}=242)$

Table 1. Characteristics and key operating figures of the participating farmers $(\mathrm{N}=242)$

\begin{tabular}{|c|c|c|}
\hline Betriebseigenschaften & Kategorie & $\%$ \\
\hline \multirow[t]{3}{*}{ Produktionsweise } & Konventionelle Landwirtschaft & 87,2 \\
\hline & Biologische Landwirtschaft & 12,4 \\
\hline & Andere & 0,4 \\
\hline \multirow[t]{6}{*}{ Betriebsgröße } & weniger als 5 ha & 0 \\
\hline & zwischen 5 und 25 ha & 9,9 \\
\hline & zwischen 26 und 50 ha & 24,4 \\
\hline & zwischen 50 und 100 ha & 39,3 \\
\hline & zwischen 100 und 500 ha & 25,6 \\
\hline & ich weiß nicht/keine Antwort & 0,8 \\
\hline \multirow{7}{*}{$\begin{array}{l}\text { Jährliches Einkommen } \\
\text { inclusive Förderung vor } \\
\text { Abzug von Steuern }\end{array}$} & weniger als $€ 10.000$,- & 5,0 \\
\hline & zwischen $€ 10.001$,- und $€ 25.000$,- & 22,7 \\
\hline & zwischen $€ 25.001$,- und $€ 50.000,-$ & 19,8 \\
\hline & zwischen $€ 50.001$,- und $€ 75.000$,- & 9,9 \\
\hline & zwischen $€ 75.001$,- und $€ 100.000$,- & 5,4 \\
\hline & mehr als $€ 100.000$,- & 2,5 \\
\hline & $\begin{array}{l}\text { weiß nicht/ich möchte } \\
\text { keine Antwort geben }\end{array}$ & 34,7 \\
\hline \multirow[t]{2}{*}{ Geschlecht } & männlich & 95 \\
\hline & weiblich & 5 \\
\hline \multirow[t]{4}{*}{ Alter } & jünger als 40 Jahre & 39,7 \\
\hline & zwischen 40 und 54 Jahre & 45 \\
\hline & zwischen 55 und 64 Jahre & 13,2 \\
\hline & 65 Jahre und älter & 2,1 \\
\hline \multirow[t]{6}{*}{ Ausbildung } & Pflichtschule & 0,8 \\
\hline & $\begin{array}{l}\text { Lehre, berufsbildende mittlere Schule } \\
\text { (z. B. Landwirtschaftliche Fachschule) }\end{array}$ & 37,6 \\
\hline & AHS/BHS & 35,1 \\
\hline & Hochschulverwandte Lehranstalt & 9,1 \\
\hline & Universität, Fachhochschule & 9,9 \\
\hline & Sonstige & 7,4 \\
\hline
\end{tabular}


Tabelle 2. Beschreibung des Standortes Engelhartstetten

Table 2. Characteristics of the experimental site Engelhartstetten

\begin{tabular}{ccc}
\hline & Einheit & Engelhartstetten \\
\hline Höhe über NN & $\mathrm{m}$ & 147 \\
Mittlerer Jahresniederschlag & $\mathrm{mm}$ & 516 \\
$(\varnothing$ 1981-2010) & & \\
Mittlere Jahrestemperatur & ${ }^{\circ} \mathrm{C}$ & 10,3 \\
$(\varnothing$ 1981-2010) & & \\
Bodentyp (FAO, 2015) & & Kalkhaltiger \\
& $\%$ & Tschernosem \\
Textur (Sand/Schluff/Ton) & & $(17,6 / 48,7 / 33,7)$ \\
pH-Wert CaCl 2 & $\%$ & 7,54 \\
Karbonatgehalt & $\%$ & 23,2 \\
Organischer Kohlenstoff & $\%$ & 2,36 \\
$\mathrm{~N}_{\text {total }}$ & $\mathrm{kg} \mathrm{ha}^{-1}$ & 0,24 \\
$\mathrm{~N}_{\text {min }} 0-90 \mathrm{~cm}$, Frühjahr 2016 & $20-32 \mathrm{~kg} \mathrm{ha}^{-1}$ \\
\hline
\end{tabular}

\subsection{Pflanzenbauliche Komponente}

Im Zuge des Projekts FATIMA konnte in zwei Jahren der Einsatz verschiedener N-Düngemengen für Winterweizen auf Probeflächen getestet werden. Feldversuche mit Winterweizen (Sorte „Саро“) wurden 2015/16 und 2016/17 am Standort „Engelhartstetten” im Marchfeld (Niederösterreich) $\left(48^{\circ} 11^{\prime} \mathrm{N}, 16^{\circ} 55^{\prime} \mathrm{E}\right)$ auf zwei benachbarten Feldern durchgeführt. Die Beschreibung des Standortes (Klima und Boden) ist in Tabelle 1 zusammengefasst. Die Bodenprobe wurde am 21.03.2016 als Mischprobe von allen Parzellen in 0-25 cm Tiefe genommen. Die in

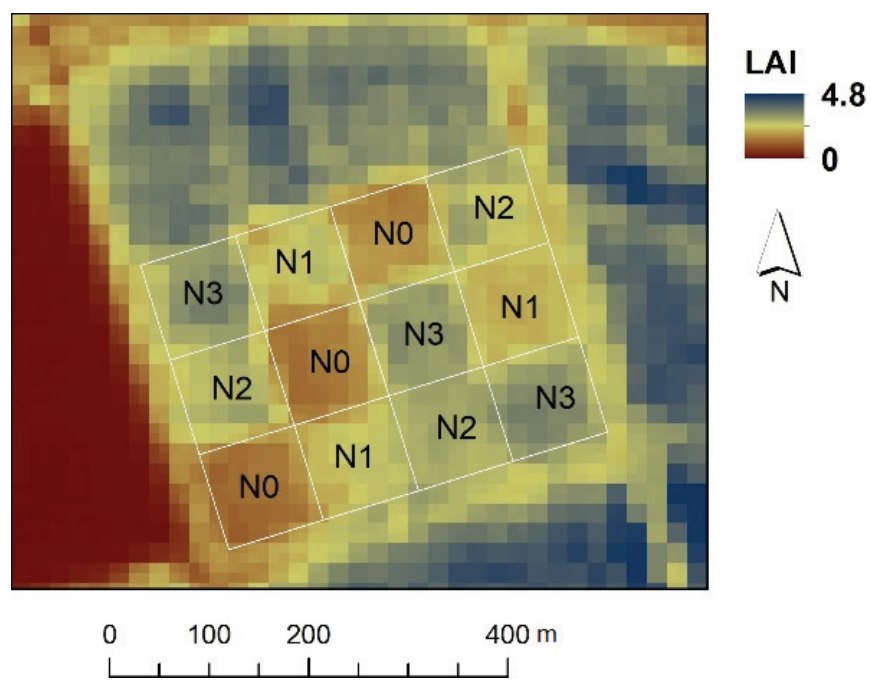

Abbildung 2. Schema des N-Düngungsversuchs in Engelhartstetten (Marchfeld, Österreich) 2016. Hintergrundbild: Sentinel-2A-Leaf-Area-Index(LAI)-Karte vom 6. Mai 2016.

Figure 2. Scheme of the $\mathrm{N}$ fertilisation experiment in Engelhartstetten (Marchfeld, Austria) 2016. Background image: Sentinel-2A Leaf Area Index (LAI) map of 6th May 2016.
Tabelle 2 angegebenen Werte entsprechen dem Mittelwert der 12 Parzellen, wenn nicht anders angegeben. Die Winterweizenversuche bestanden neben der Nulldüngungsvariante N0 (0 kg N ha-1) aus drei unterschiedlichen Düngungsstufen mit folgenden Gesamtdüngungsmengen (N1: $60 \mathrm{~kg} \mathrm{~N} \mathrm{ha}{ }^{-1}$; N2: $120 \mathrm{~kg} \mathrm{~N} \mathrm{ha}^{-1}$; N3: $180 \mathrm{~kg} \mathrm{~N} \mathrm{ha}^{-1}$; Abbildung 5), die als Kalkammonsalpeter ausgebracht und gleichmäßig auf drei Düngungstermine im März, April und Mai aufgeteilt wurden. Die Feldversuche bestanden aus einem randomisierten Blockdesign mit drei Wiederholungen, wobei die Parzellen jeweils $100 \mathrm{~m} \times 100 \mathrm{~m}$ maßen. Ein Beispiel für die Anlage der Feldversuche (Winterweizen im Jahr 2015/16) ist in Abbildung 2 dargestellt. Die Phosphor- und Kaliumdüngung wurde entsprechend den Richtlinien für die sachgerechte Düngung (BMLFUW, 2017) durchgeführt. Als Pflanzenschutzmaßnahme wurden 1,2 1 ha $^{-1}$ des Herbizids Tomigan XL jeweils im April ausgebracht. Die Bewirtschaftungsdaten der Anbauperioden 2015/16 und 2016/17 sind in Tabelle 2 dargestellt.

Der Feldversuch diente einerseits der Validierung der Satellitendaten und wurde darüber hinaus als Basis für die Bewertung der Wirtschaftlichkeit der unterschiedlichen N-Düngemengen herangezogen. Unter Berücksichtigung der Kosten für Dünger, Arbeitszeit, Maschineneinsatz, den Marktpreisen für Weizen im Juni der beiden Jahre und dem mit dem eingesetzten N-Dünger erwirtschafteten Ertrag und den erzielten Qualitäten wurde eine vereinfachte ökonomische Analyse der Wirtschaftlichkeit der Düngemaßnahmen durchgeführt (Eq.1):

\section{Ertrag $\left[\mathrm{kg} \mathrm{t}^{-1}\right] \times$ erzielter Getreidepreis für entsprechende Qualität [ $\left.€ \mathrm{t}^{-1}\right]$ - Düngerkosten $\left[€ \mathrm{ha}^{-1}\right]$ \\ - Arbeits- und Maschineneinsatz für Düngung [ $\left.€ \mathrm{ha}^{-1}\right]$ = Umsatz abzüglich der variablen Stickstoffmanagementkosten $\left[€ \mathrm{ha}^{-1}\right]$}

Tabelle 3. Bewirtschaftungsdaten der Anbauperioden 2015/16 und 2016/17 Table 3. Cultivation dates of the seasons 2015/16 and 2016/17

\begin{tabular}{ccc}
\hline Anbauperiode & $\mathbf{2 0 1 5 / 2 0 1 6}$ & $\mathbf{2 0 1 6 / 2 0 1 7}$ \\
\hline Bodenbearbeitung & November 2015 & 9. und 11. November 2016 \\
Saat & 17. November 2015 & 15. November 2016 \\
1. N-Düngung & 24. März 2016 & 16. März 2017 \\
2. N-Düngung & 21. April 2016 & 25. April 2017 \\
3. N-Düngung & 19. Mai 2016 & 30. Mai 2017 \\
Ernte & 26. Juli 2016 & 20. Juli 2017 \\
\hline
\end{tabular}


Die Daten stammen von den Webseiten der AgrarMarktAustria AMA (Kalkammonsalpeterpreise) und der Landwirtschaftskammer Niederösterreich (Getreidepreise) sowie aus eigenen Quellen. Die Angaben des Vergleichsbetriebs aus dem Marchfeld wurden mit Daten aus dem KTBL-Feldarbeitsrechner (https://daten.ktbl.de) verglichen und auf Plausibilität geprüft. Für die Studie wurden aufgrund der regionalen Berechnung die österreichischen Daten herangezogen. Detaillierte Angaben finden sich in Tabelle 3.

\subsection{Technische Komponente}

Die technische Komponente der Studie umfasste die Auswertung von Satellitendaten, um einerseits die wissenschaftlichen Aspekte der Projektaktivitäten zu unterstützen (Vuolo et al., 2017) und andererseits ein praktisch einsetzbares Werkzeug für die N-Düngung zu entwickeln. Das entwickelte web-basierte Tool wird nachfolgend behandelt. Für die praktische Anwendung wurden Sentinel-2-Daten verwendet, um die zeitliche Entwicklung des Blattflächenindex (Leaf Area Index LAI) während der gesamten Vegetationsperiode zu erfassen. Der LAI korreliert stark mit der vorhandenen Biomasse (Beadle et al., 1985) und die zeitliche Entwicklung des LAI gibt Aufschluss über die Produktivität der Kulturen unter den gegebenen Wachstumsbedingungen.

Die Satellitendaten und die LAI-Karten wurden aus dem Sentinel-2-Portal des Instituts für Geomatik der Universität für Bodenkultur bezogen (Vuolo et al., 2016). Die verarbeiteten Daten wurden automatisch in einer Datenbank gespeichert. Die potenzielle Produktivität wurde aus Zeitreihen des LAI gewonnen. Die LAI-Daten werden zunächst geglättet und die Lücken gefüllt, um regelmäßig verteilte LAI-Zeitreihen (7-Tage-Intervall) zu erhalten. Anschließend wurden die LAI-Daten zeitlich vom Beginn bis zum Ende der Vegetationsperiode für jedes Jahr einzeln bestimmt und dann ein z-Wert für jedes Feld berechnet. Basierend auf dem LAI wurde ein einheitsloser Indikator für die potenzielle Produktivität im Bereich von 0-255 errechnet und für eine Optimierung der N-Düngung verwendet.

Für die Erstellung der N-Applikationskarten standen sowohl einzelne Satellitenbildaufnahmen als auch die historischen Produktivitätskarten zur Verfügung. Für die zukünftige Anwendung können daher je nach Einsatzzeitpunkt aktuelle oder historische Karten für die optimierte $\mathrm{N}$-Düngung verwendet werden.

\section{Ergebnisse und Diskussion}

\subsection{Sozio-ökonomische Komponente}

Die TeilnehmerInnen wurden nach den für sie zurzeit größten Herausforderungen des Landwirtschaftssektors befragt. Die Marktbedingungen (1) wurden am öftesten genannt, gefolgt von den hohen bürokratischen Anforderungen (2) und extremen Wetterereignissen (3). Im Mittelfeld finden sich Themen mit Bezug zu Ressourcenverfügbarkeit wie Zugang zu Wasser (4) und Land (5). Umweltthemen, wie z. B. Bodendegradation und Stickstoffanreicherung im Grundwasser, wurden nur von wenigen LandwirtInnen als direkte Herausforderungen für den Betrieb angesehen. Dennoch betrachten es die LandwirtInnen als Notwendigkeit, die Umwelt zu schützen und Maßnahmen für Umweltschutz am Betrieb zu treffen.

Um herauszufinden, ob eine generelle Innovations- und Investitionsbereitschaft besteht, wurden die UmfrageteilnehmerInnen nach ihren Investitionen innerhalb der letzten fünf Jahre gefragt. Zwei Drittel der Befragten hatten sowohl in verbessertes Management (z. B. Farm Management

Tabelle 4. Übersicht über die Komponenten der Wirtschaftlichkeitsberechnung

Table 4. Overview of the components of the profitability calculation

\begin{tabular}{|c|c|c|c|c|}
\hline Komponente & Preis 2016 & Preis 2017 & Einheit & Quelle \\
\hline Kalkammonsalpeter $27 \% \mathrm{~N}$ & 25,79 & 19,05 & $€ 100 \mathrm{~kg}^{-1}$ & AMA Marktbericht (2016) und AMA Marktbericht (2017) \\
\hline Getreidepreise (jeweils Durchschnitt) & & & $€ \mathrm{t}^{-1}$ & Börse für landwirtschaftliche Produkte in Wien \\
\hline Premiumweizen (> $15 \%$ Proteingehalt) & kein Wert & 190 & & \\
\hline $\begin{array}{l}\text { Qualitätsweizen } \\
\text { (14-15\% Proteingehalt) }\end{array}$ & 154,5 & 171,5 & & $\begin{array}{l}\text { Kursblatt Nr. } 24 \text { vom 15. Juli } 2016 \\
\text { Kursblatt Nr. } 24 \text { vom 14. Juni } 2017\end{array}$ \\
\hline $\begin{array}{l}\text { Mahlweizen } \\
\text { (12,5-14 \% Proteingehalt) }\end{array}$ & 145 & 154 & & \\
\hline $\begin{array}{l}\text { Futterweizen } \\
(<12,5 \% \text { Proteingehalt })\end{array}$ & 137,5 & 151,5 & & \\
\hline $\begin{array}{l}\text { Arbeits- und Maschineneinsatz (ohne Dünger): } \\
\text { Maschinen- und Arbeitszeit }\end{array}$ & 10,67 & 10,67 & $€ \mathrm{ha}^{-1}$ & Erhobener Wert von Vergleichsbetrieb aus dem Marchfeld \\
\hline
\end{tabular}


Software) als auch in bessere technische Ausrüstung investiert. Immerhin $40 \%$ konnten des Weiteren auf geänderte Methoden unter Berücksichtigung von Umweltaspekten im Bereich ihrer landwirtschaftlichen Praxis verweisen (z. B. Umstieg auf biologische Wirtschaftsweise oder reduzierte Bodenbearbeitung). Diese Werte zeigen eine generell hohe Innovationsbereitschaft der TeilnehmerInnen im Vergleich zu den Umfragewerten in Italien, wo die Umfrage ebenfalls durchgeführt wurde (Blasch et al., 2020).

Die Bereitschaft der TeilnehmerInnen, die bereits vorgestellten Optionen (voll-, teilweise oder nicht automatisiertes N-Düngemanagement) auf ihrem Betrieb einzusetzen, wurde in einem Choice-Experiment abgefragt.

Alle drei Formen der teilflächenspezifischen N-Düngung, die vorgestellt wurden, waren den LandwirtInnen bekannt. Die Hälfte der Befragten gab an, den Erwerb von teilweise (51\%) oder nicht (47\%) automatisierten Technologien bereits in Erwägung gezogen zu haben. Das Interesse für die voll automatisierte Lösung war mit $14 \%$ weniger stark ausgeprägt.

Die Auswertung des Choice-Experiments ergab, dass $75 \%$ der LandwirtInnen generell bereit dazu sind, Technologien, die der teilflächenspezifischen Ausbringung von Stickstoff dienen, anzuwenden. Die TeilnehmerInnen haben allerdings unterschiedliche Präferenzen bezüglich der einzelnen vorgeschlagenen Technologien. Die voll automatische Sensorlösung wurde von der Mehrheit der TeilnehmerInnen abgelehnt. Bei den anderen beiden Technologiegruppen ergab sich ein differenziertes Bild.

Das Choice-Experiment zeigte, dass Technologieoptionen mit höheren Erträgen und Düngereinsparpotential von den LandwirtInnen nicht durchgehend bevorzugt wurden, jedoch eine persönliche Beratung sowie eine potenzielle Verbesserung der Grundwasserqualität einen signifikant positiven Einfluss auf die Investitionsbereitschaft haben. Auch die Kosten der Technologie spielen eine Rolle bei der Anschaffung. Ein hoher Anschaffungspreis stößt erwartungsgemäß auf Ablehnung. Auch die Größe des Betriebs hatte Einfluss auf die Auswahl. Größere Betriebe sind eher an teil- bzw. vollautomatisierten Systemen interessiert.

Eine weitere Beobachtung konnte gemacht werden: Konventionell wirtschaftende LandwirtInnen sind an teilflächenspezifischer Ausbringung interessierter als biologisch arbeitende LandwirtInnen. Dies kann auf die limitierten technischen Möglichkeiten der Ausbringung von biologischem Dünger, wie zum Beispiel Gülle, sowie auf alternative Stickstoffversorgung über Leguminosen, zurückgeführt werden.
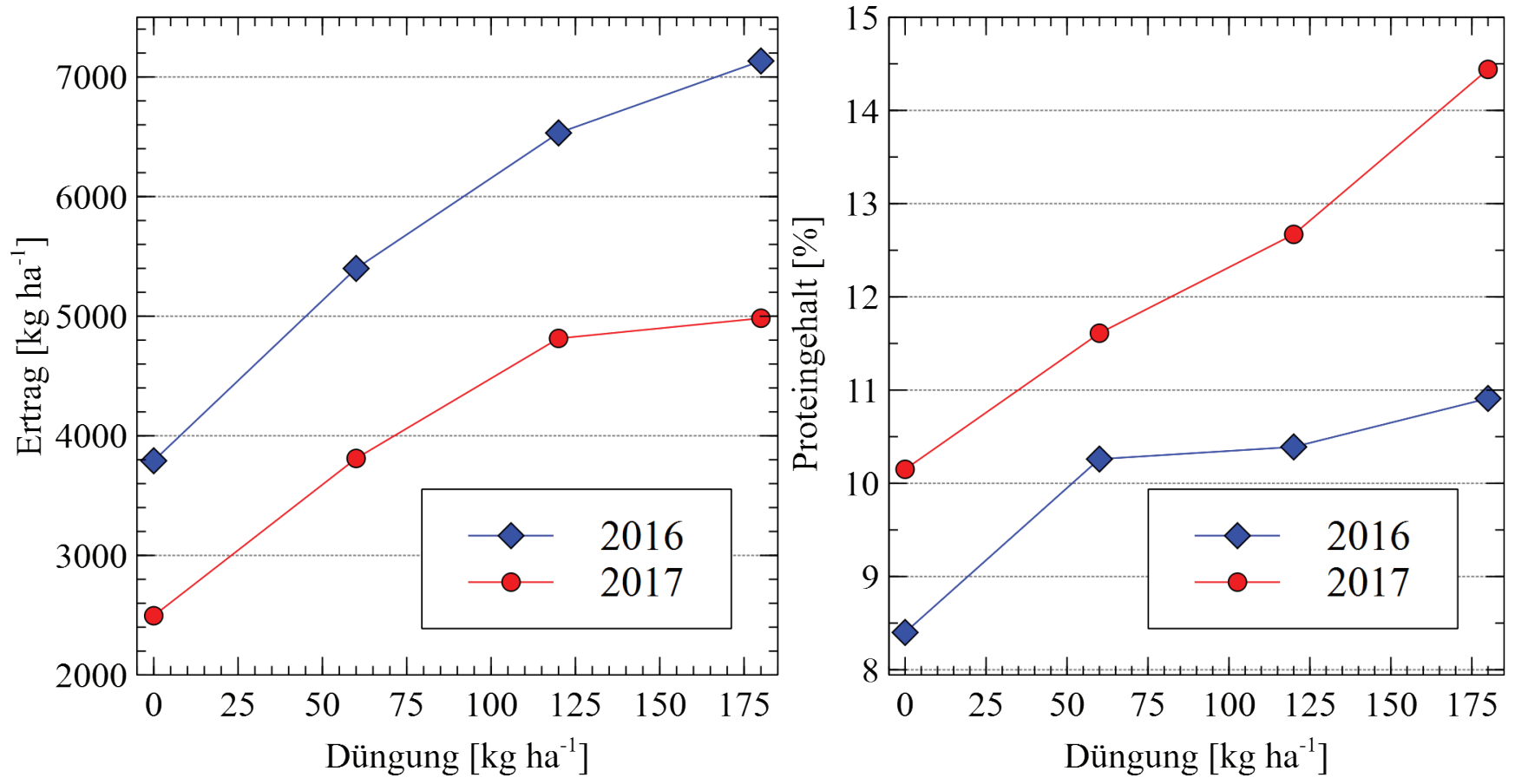

Abbildung 3. Weizenertrag (links) und Proteingehalt (rechts) der vier Düngestufen.

Figure 3. Wheat yield (left) and protein content (right) of the four fertilisation levels. 


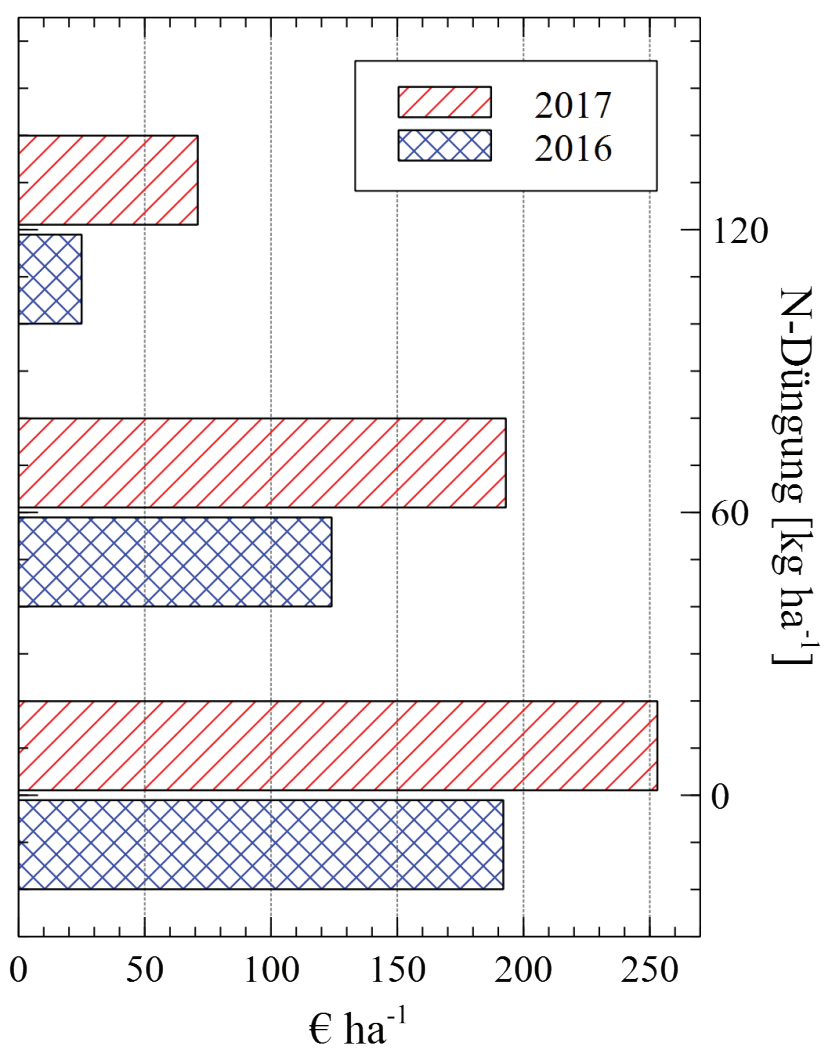

Abbildung 4. Einkommenseinbußen aufgrund verringerter N-Düngung im Vergleich zu $180 \mathrm{~kg} \mathrm{~N} \mathrm{ha}^{-1}$.

Figure 4. Loss of income due to reduced $\mathrm{N}$ fertilisation compared to $180 \mathrm{~kg} \mathrm{~N} \mathrm{ha}^{-1}$.

\subsection{Pflanzenbauliche Komponente}

Das Ziel von Düngemaßnahmen ist vorrangig die Erzielung eines höheren Ertrags und einem daraus resultierenden höheren Erlös. Die FATIMA-Versuchsflächen sind Grundlage für die Untersuchung der Wirtschaftlichkeit von variierenden $\mathrm{N}$-Düngemengen in Winterweizen.

Beim Vergleich von Stickstoffzufuhr und Ertrag zeigte sich, dass ab einem gewissen Versorgungsgrad die Kurve des Ertragszuwachses mit steigender N-Düngemenge abflacht (Abbildung 3).

Die ökonomische Analyse zeigt den folgenden Trend (Abbildung 4): Beim gänzlichen Verzicht auf Stickstoffdünger bewegen sich die Einkommenseinbußen im Vergleich zur höchsten Stufe im Bereich von 192-253€ ha ${ }^{-1}$, sind also durchaus beträchtlich. Anders zeigt sich die Situation beim Vergleich von $120 \mathrm{~kg} \mathrm{~N} / \mathrm{ha}$ mit $180 \mathrm{~kg} \mathrm{~N} \mathrm{ha}^{-1}$. Die Unterschiede liegen im Bereich von $25-71 € \mathrm{ha}^{-1}$, wobei sich besonders 2016 aufgrund niedriger Abnahmeund höherer Stickstoffpreise der erhöhte Düngereinsatz kaum auszahlte. Eine Untersuchung der Energieeffizienz der verschiedenen Düngeoptionen des Feldversuches von Moitzi et al. (2020) kommt zu dem Ergebnis, dass diese ab $120 \mathrm{~kg} \mathrm{~N}^{-1}$ abnimmt. Die Daten basieren auf der Evaluierung der Versuchsflächen und können daher kein allgemeingültiges Bild geben. Dennoch zeigen die Ergebnisse, dass der höhere N-Düngereinsatz nicht mit entsprechend höherem Einkommenszuwachs gleichzusetzen ist. Des Weiteren machen die Ergebnisse deutlich, dass externe Einflussfaktoren eine große Rolle spielen. So unterschieden sich Witterungsbedingungen und die aktuellen Marktpreise von Dünger und Weizen in den beiden Jahren erheblich. Im Jahr 2016 betrug der Niederschlag von Jänner bis Mai 256 mm, während es im Jahr 2017 nur $164 \mathrm{~mm}$ waren (ZAMG, 2017).

\subsection{Technische Komponente}

Abbildung 5 (rechts) zeigt einen Ausschnitt der Produktivitätskarte, die für ganz Österreich erstellt wurde. Die Karte zeigt deutlich die räumlichen Muster, die für die Variabilität der Böden in Ostösterreich typisch ist. Grüne Zonen sind Zonen hoher Produktivität, rote Zonen weisen niedriges Biomassewachstum aus. Ähnliche Strukturen sind auch auf Orthofotos in Echtfarbe der Region zu erkennen (links). Eine Validierung mit der Österreichischen Bodenkarte war aufgrund der großen Unterschiede in der Auflösung der beiden Karten nicht möglich. Daher wurde als Alternative eine Validierung auf Feldebene für zwei Felder vorgenommen. Abbildung 6 zeigt den Vergleich zwischen den Werten der Produktivitätskarte und den Erntemengen der betreffenden Punkte für zwei Felder, die von LandwirtInnen der Region unter konventionellen Bedingungen bewirtschaftet wurden und deren mittels Drescher mit integriertem Wiegesystem ermittelten Ertragsdaten dem Projektteam zur Verfügung gestellt wurden. Die Ergebnisse zeigen eine positive Korrelation des Ertragspotenzials mit dem tatsächlich erzielten Ertrag für beide Felder. Weitere Validierungsarbeiten werden notwendig sein, um zukünftig noch aussagekräftigere Resultate zu erzielen.

Die Produktivitätskarte wurde Testnutzern in einer Online-Anwendung (Abbildung 7) zur Verfügung gestellt. Der Nutzer kann zwischen dem aktuellen Satellitenbild oder der auf historischen Bildern basierenden Produktivitätskarte wählen, um die Zonen für teilflächenspezifische N-Düngung zu definieren.

Die notwendigen Eingabeparameter sind die Mindestfläche der einzelnen Zonen, die Unter- und Obergrenze der Stickstoffgabe sowie die gewählte Düngestrategie. 

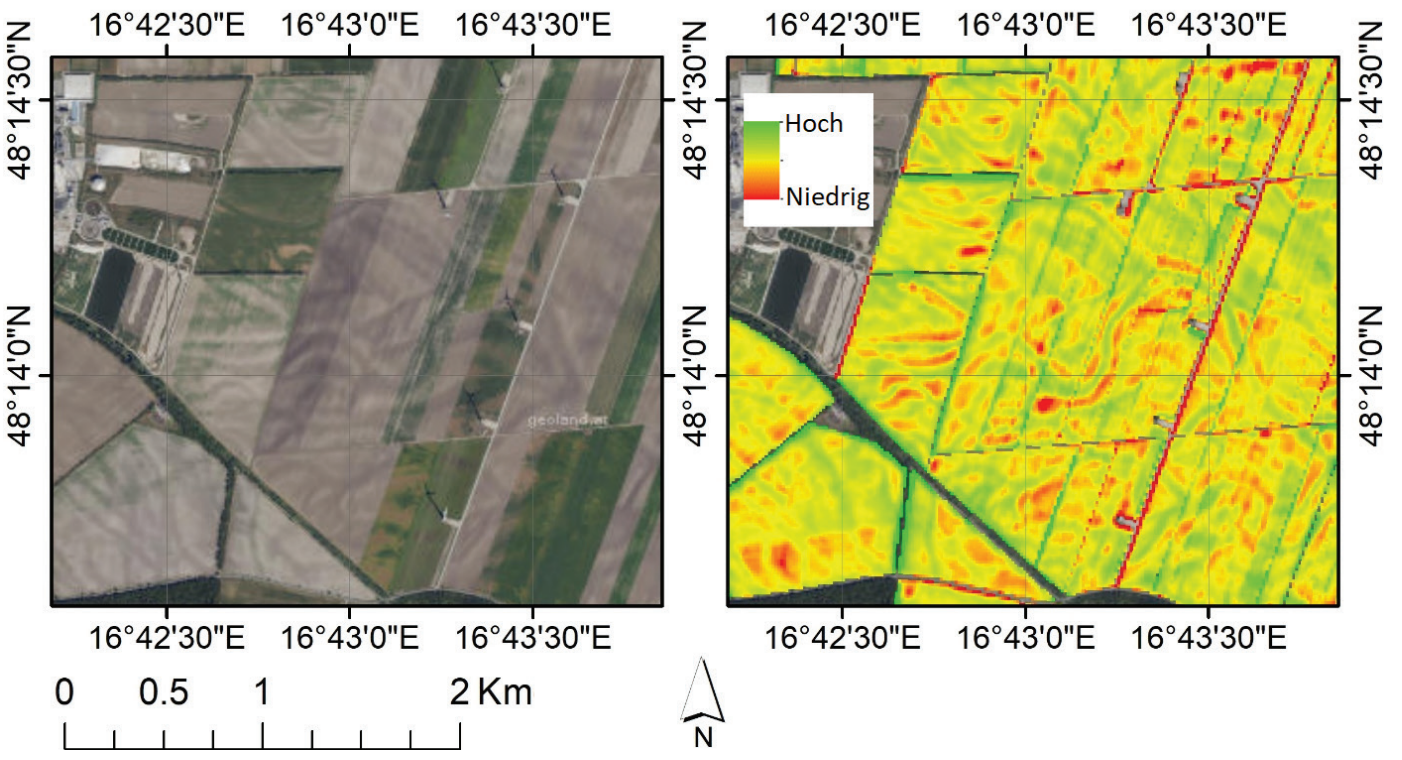

Abbildung 5. Vergleich zwischen der Produktivitätskarte (rechts) und einem hochaufgelösten Geoland-Basemap-Orthofoto (RGB, Echtfarbe) (links) in einem landwirtschaftlich genutzten Gebiet im Marchfeld.

Figure 5. Comparison between the productivity map (right) and a high-resolution Geoland Basemap orthophoto (RGB, true color) (left) in an agricultural area in Marchfeld.

Welche Datengrundlage für die Erstellung der Karte herangezogen wird, entscheiden die NutzerInnen. Wichtig ist hier insbesondere die aktuelle Pflanzenentwicklung. Typischerweise erfolgt bei Wintergetreide die erste Düngergabe vor dem Auflaufen der Pflanze. Zu diesem Zeitpunkt ist noch keine räumliche Variabilität der Biomasse sichtbar. In diesem Fall wird die historische Produktivitätskarte für die Erstellung der Applikationskarte herangezogen. Für die zweite und dritte Düngung können dagegen aktuelle Satellitenbilder verwendet werden, die den aktuellen Wachstumszustand darstellen. Zwei verschiedene Strategien sind für die teilflächenspezifische Düngung möglich: mehr Dünger in weniger entwickelten Zonen (catch $u p$ ) oder mehr Dünger in besser entwickelten Zonen (top up).
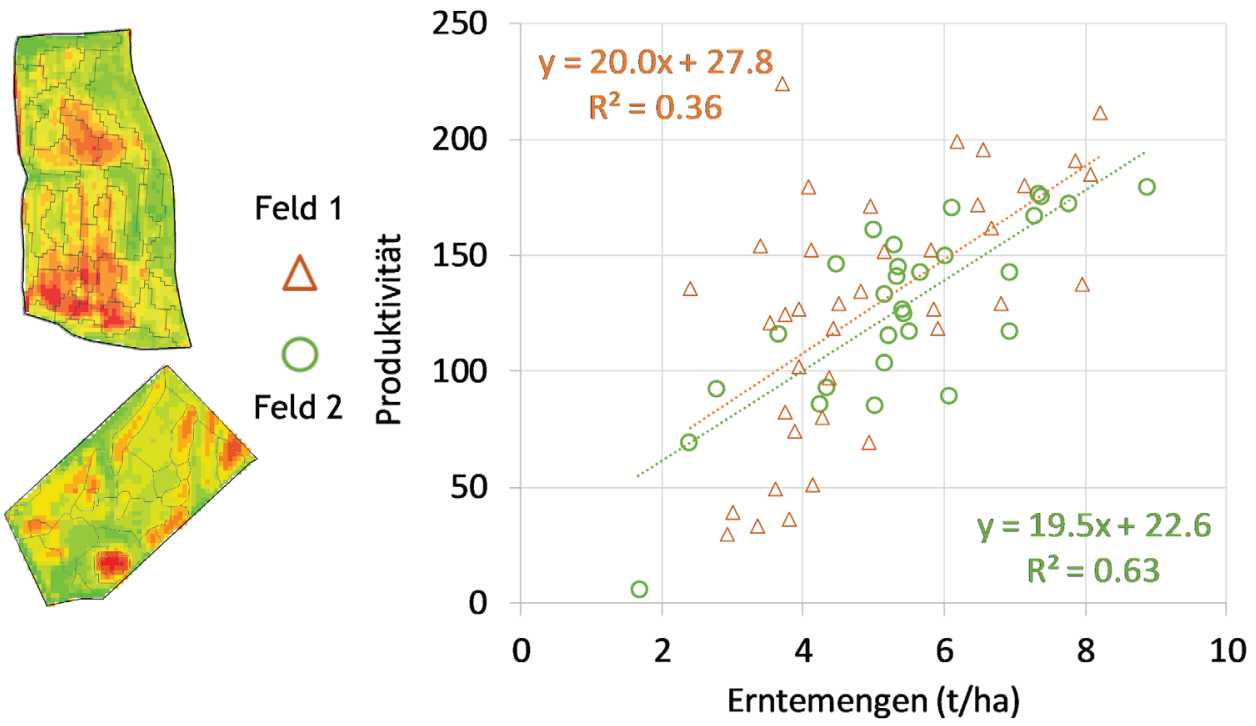

Abbildung 6. Erntemengen vs. Produktivität für zwei Felder im Jahr 2017. Beide Datenquellen wurden für die einzelnen Managementzonen aggregiert. Jeder Punkt in der Abbildung steht für die Daten einer Managementzone.

Figure 6. Yields vs. productivity for two fields in 2017 , both data sources were aggregated for the individual management zones. Each point in the figure represents the data for one management zone. 
Abbildung 7. Auszug aus der Online-Anwendung für die Kartenerstellung für die teilflächenspezifische N-Düngung.

Figure 7. Screenshot of the online application for the creation of maps for variable-rate nitrogen fertilisation.

Copy to clipboard CSV Excel Shapefile John Deere Trimble TXT GRD

Step 1 - Define management zones

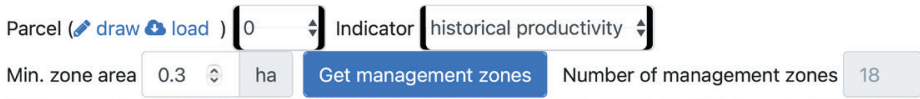

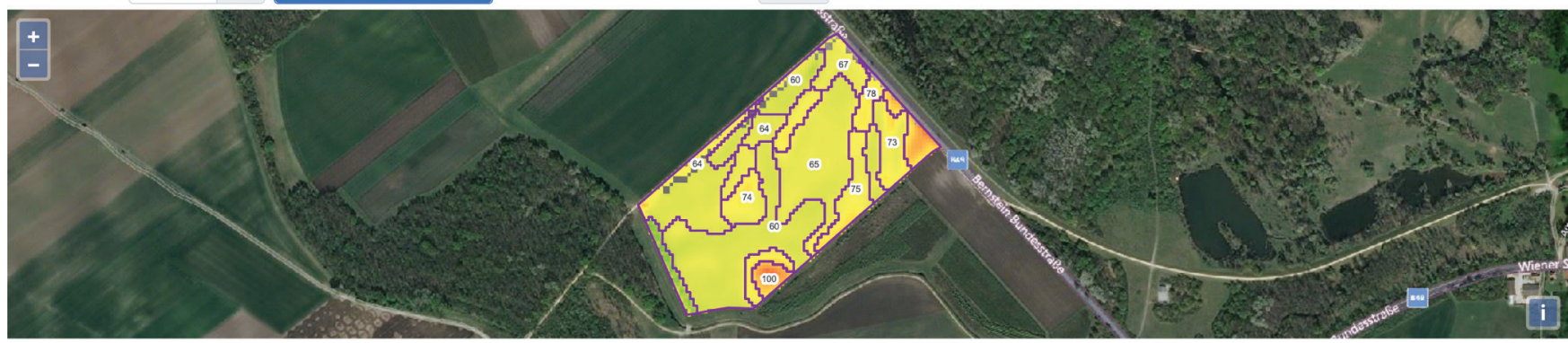

Step 2 - Modulate fertilization amounts

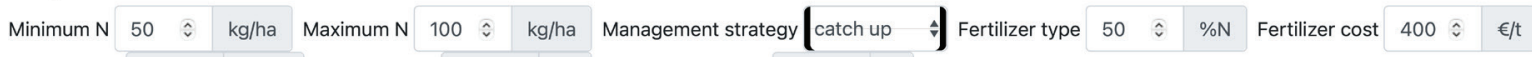

Avg nitrogen $66 \approx \mathrm{kg} / \mathrm{ha}$ Total fertilizer $1950 \approx \mathrm{kg} \quad$ Total fertilizer cost $780 \approx €$

Step 3 - Detailed amounts

\begin{tabular}{|c|c|c|c|}
\hline Zone No $\uparrow$ & Area $\uparrow \downarrow$ & Fertilizer Rate $\uparrow$ & Nitrogen Rate $\uparrow \downarrow$ \\
\hline 1 & 0.3 ha & $118 \mathrm{~kg} / \mathrm{ha}$ & $59 \hat{\vee} \mathrm{kg} / \mathrm{ha}$ \\
\hline 2 & $0.3 \mathrm{ha}$ & $136 \mathrm{~kg} / \mathrm{ha}$ & $68 \hat{\imath} \mathrm{kg} / \mathrm{ha}$ \\
\hline 3 & 0.31 ha & $200 \mathrm{~kg} / \mathrm{ha}$ & $100 \hat{\imath} \mathrm{kg} / \mathrm{ha}$ \\
\hline
\end{tabular}

In einem letzten Schritt können die Daten vom Endnutzer im benötigten Datenformat heruntergeladen werden und dann direkt an das Traktorterminal für eine teilflächenspezifische Düngung übertragen werden.

\section{Schlussfolgerungen}

Die Ergebnisse des Forschungsprojektes beleuchten das Potential einer teilflächenspezifischen Düngung aus verschiedenen Perspektiven.

Bei den LandwirtInnen selbst ist die Technologie zumindest bekannt und das Interesse für die Anwendung groß. Die Verwendung der vorgestellten Produktivitätskarten ist weder teuer noch kompliziert. Auch wenn der Traktor nicht mit GPS und Bordcomputer ausgestattet ist, können LandwirtInnen die Karten auf ihre mobilen Endgeräte laden und bei der Fahrt über das Feld manuell mehr oder weniger Dünger ausbringen. Diese Applikation birgt besonders für kleine und mittelgroße Betriebe, die nicht in Sensortechnologie investieren wollen, großes Potenzial.
Die wirtschaftliche Evaluierung der verschiedenen Düngevarianten der Versuchsflächen ergab, dass aufgrund des Gesetzes des abnehmenden Ertragszuwachses besonders die hohen Düngergaben von $180 \mathrm{~kg} \mathrm{ha}^{-1} \mathrm{zu}$ überdenken sind. Anstatt einheitlichen Managements können in Zukunft mithilfe der Produktivitätskarten die ausgebrachten Stickstoffmengen innerhalb eines Felds variiert werden, um Stickstoff nur dort auszubringen, wo er benötigt wird.

Ein direkter Vergleich der verschiedenen Düngestrategien "catch up" und "top up" wäre sowohl aus ökonomischer als auch ökologischer Sicht interessant und bietet sich für weiterführende Untersuchungen an, um einerseits die ökonomischen Vorteile der teilflächenspezifischen Düngung zu quantifizieren andererseits aber auch das Risiko von erhöhten Nitratwerten im Grundwasser bei verstärkter Düngung wachstumsschwächerer Zonen abzuklären.

Des Weiteren werden sich die zukünftigen Aktivitäten auf eine Validierung und eine leichtere Anwendbarkeit der Daten konzentrieren, um die Informationen unter möglichst vielen AnwenderInnen zu verbreiten. Dies entspricht auch dem Grundgedanken der neuen Gemeinsamen 
Agrarpolitik (GAP) der Europäischen Union, die sich von einem regelgeleiteten System zu einem leistungs- und ergebnisbasierten Ansatz entwickeln möchte und mit der GAP 2021 verpflichtende Werkzeuge zum verbesserten Nährstoffmanagement einführen wird (Europäische Union, 2019).

\section{Danksagung}

Die Studie wurde im Rahmen des Projektes „FATIMA“ (FArming Tools for external nutrient Inputs and water MAnagement, http://fatima-h2020.eu/) durchgeführt. Das Projekt wurde vom Programm Horizon 2020 der Europäischen Union (GA Nr. 633945) finanziert. Wir bedanken uns bei allen Personen, die als InterviewerInnen bei den Befragungen tätig waren.

\section{Literaturverzeichnis}

AMA Marktbericht (2016): https://www.ama.at/get attachment/080f3742-9b1b-44f8-95a2-af62b2ecc225/ Marktbericht_Getreide_Oelsaaten_02_2016.pdf. Abgerufen am 21.04.2021.

AMA Marktbericht (2017): https:/www.ama.at/getattachment/ 7d0b4c66-28b6-448e-b667-6f19b0217210/Marktbericht_ Getreide_Oelsaaten_02_2017.pdf, abgerufen am 21.04.2021

AMA (2018): INVEKOS Schläge Österreich 2018. https://www.data.gv.at/katalog/dataset/f7691988-e57c4ee9-bbd0-e361d3811641. Abgerufen am 27.05.2021.

Amt der NÖ Landesregierung (2016): Der Grüne Bericht 2016. St. Pölten, https:/gruenerbericht.at/cm4/jdownload/send/10-gr-bericht-niederrreich/1879-niederoesterreich-gb-2016. Abgerufen am 21.04.2021.

Beadle, C. L. und S. P. Long (1985): Photosynthesis - Is it limiting to biomass production? Biomass 8(2), 119-168.

Blasch, J., van der Kroon, B., van Beukering, P., Munster, R., Fabiani, S., Nino, P. und S. Vanino (2020): Farmer preferences for adopting precision farming technologies: A case study from Italy. European Review of Agricultural Economics, jbaa031.

BMLFUW (2010): Verordnung des Bundesministers für Land-und Forstwirtschaft, Umwelt und Wasserwirtschaft über den guten chemischen Zustand des Grundwassers (Qualitätszielverordnung Chemie Grundwasser - QZV Chemie GW),StF: BGBl. II Nr. 98/2010 [CELEX-Nr:
31991L0692, 32006L0118] Bundesministerium für Land- und Forstwirtschaft, Umwelt und Wasserwirtschaft, Wien. https://www.ris.bka.gv.at/GeltendeFassung/Bundesnormen/20006738/QZV\%20Chemie\%20 GW\%2c\%20Fassung\%20vo m \% 2030.08.2019. pdf?FassungVom=2019-08-30. Abgerufen am 27.05.2021.

BMLFUW (2017): Richtlinien für die sachgerechte Düngung im Ackerbau und Grünland - Anleitung zur Interpretation von Bodenuntersuchungsergebnissen. 7. Auf., Fachbeirat für Bodenfruchtbarkeit und Bodenschutz, Bundesministerium für Land- und Forstwirtschaft, Umwelt und Wasserwirtschaft, Wien.

BörsefürlandwirtschaftlicheProduktein Wien(2016):KursblattNr.24vom 15.Juli2016.https://www.boersewien.at/ ?id=10200\&api=kursblatt.pdf\&kursblatt=24_2016. Abgerufen am 21.04.2021.

BörsefürlandwirtschaftlicheProduktein Wien(2017):Kursblatt Nr. 24 vom 14. Juni 2017. https://www.boersewien. at $/$ id=10200\&api=kursblatt.pdf\&kursblatt=24_2017. Abgerufen am 21.04.2021.

Cameron, K. C., Di, H. J. und J. L. Moir (2013): Nitrogen losses from the soil/plant system: A review: Nitrogen losses. Annals of Applied Biology 162(2), 145-173. Europäische Kommission (2019): Die Gemeinsame Agrarpolitik nach 2020: Umweltnutzen und Vereinfachung. https://ec.europa.eu/info/sites/default/files/food-farming-fisheries/key_policies/documents/cap-post2020-environ-benefits-simplification_de.pdf. Abgerufen am 27.05.2021.

FAO (2014): World reference base for soil resources 2014: International soil classification system for naming soils and creating legends for soil maps. Food and Agriculture Organisation, Rome, Italy.

Galloway, J. N., Aber, J. D., Erisman, J. W., Seitzinger, S. P., Howarth, R. W., Cowling, E. B. und B. J. Cosby (2003): The Nitrogen Cascade. BioScience 53(4), 341-356.

Groher, T., Heitkämper, K., Walter, A., Liebisch, F. und C. Umstätter (2020): Status quo of adoption of precision agriculture enabling technologies in Swiss plant production. Precision Agriculture 21(6), 1327-1350.

Hansen, B., Thorling, L., Schullehner, J., Termansen, M. und T. Dalgaard (2017): Groundwater nitrate response to sustainable nitrogen management. Scientific Reports 7(1), 8566.

Hawkesford, M. J. (2014): Reducing the reliance on nitrogen fertilizer for wheat production. Journal of Cereal Science 59(3), 276-283. 
Heege, H. J. (Hrsg.) (2013): Precision in Crop Farming. Springer Netherlands. Site Specific Concepts and Sensing Methods: Applications and Results. Springer, Dordrecht, The Netherlands.

Juntakut, P., Haacker, E. M. K., Snow, D. D. und C. Ray (2020): Risk and Cost Assessment of Nitrate Contamination in Domestic Wells. Water 12(2), 428.

Lewandowski, A. M., Montgomery, B. R., Rosen, C. J. und J. F. Moncrief (2008): Groundwater nitrate contamination costs: A survey of private well owners. Journal of Soil and Water Conservation 63(3), 153-161.

Moitzi, G., Spiegel, H., Sandén, T., Vuolo, F., Essl, L., Neugschwandtner, R. W. und H. Wagentristl (2020): Energieeinsatz und Energieeffizienz von Winterweizen bei unterschiedlicher mineralischer Stickstoffdüngung im Marchfeld. Die Bodenkultur: Journal of Land Management, Food and Environment 71(2), 55-67.

Nachtnebel, H. P., Fuchs, M., Schober, S. und K. Hebenstreit (2001): Die Hydrologie Österreichs unter dem Einfluß von Szenarien einer möglichen Klimaänderung, Endbericht Teil 1. Auftraggeber: Bundesministerium für Wissenschaft und Verkehr und Bundesministerium für Umwelt, Jugend und Familie, Wien.

Neugebauer, N. und F. Vuolo (2014): Crop water requirements on regional level using remote sensing data - A case study in the Marchfeld Region. Photogrammetrie, Fernerkundung, Geoinformation 5, 369-381.

Rechnungshof (2018): Bericht des Rechnungshofes: Umsetzung der Wasserrahmenrichtlinie im Bereich Grundwasser im Weinviertel; Follow-up-Überprüfung. Reihe BUND 2018/63, https://www.rechnungshof.gv.at/rh/home/home/home_7/Wasserrahmenrichtlinie_FuP.pdf. Abgerufen am 21.04.2021.

Rosenstock, T. S., Liptzin, D., Dzurella, K., Fryjoff-Hung, A., Hollander, A., Jensen, V., King, A., Kourakos, G., McNally, A., Pettygrove, G. S., Quinn, J., Viers, J. H., Tomich, T. P. und T. Harter (2014): Agriculture's Contribution to Nitrate Contamination of Californian Groundwater (1945-2005). Journal of Environmental Quality 43(3), 895-907.

Snyder, C. S., Bruulsema, T. W., Jensen, T. L. und P. E. Fixen (2009): Review of greenhouse gas emissions from crop production systems and fertilizer management effects. Reactive nitrogen in agroecosystems: Integration with greenhouse gas interactions. Agriculture, Ecosystem \& Environment 133(3), 247-266.

Statistik Austria (2016): Agrarstrukturerhebung 2016. Statistik Austria StatCube. www.statcube.at. Abgerufen am 27.05.2021.

Umweltbundesamt (2016): Elfter Umweltkontrollbericht. Wien, https://www.umweltbundesamt.at/filead$\mathrm{min} /$ site/publikationen/REP0600BF.pdf. Abgerufen am 21.04.2021.

Umweltbundesamt (2017): Wieviel zahlen Trinkwasserkunden für die Überdüngung. Factsheet, https://www. umweltbundesamt.de/sites/default/files/medien/2546/ dokumente/factsheet_kosten_nitrat_trinkwasser_0.pdf. Abgerufen am 21.04.2021.

Vuolo, F., Neuwirth, M., Immitzer, M., Atzberger, C. und W.-T. Ng (2018): How much does multi-temporal Sentinel-2 data improve crop type classification? International Journal of Applied Earth Observation and Geoinformation 72, 122-130.

Vuolo, F., Essl, L., Zappa, L., Sandén, T. und H. Spiegel (2017): Water and nutrient management: The Austria case study of the FATIMA H2020 project. Advances in Animal Biosciences 8(2), 400-405.

Vuolo, Francesco, Żółtak, M., Pipitone, C., Zappa, L., Wenng, H., Immitzer, M., Weiss, M., Baret, F. und C. Atzberger (2016): Data Service Platform for Sentinel-2 Surface Reflectance and Value-Added Products: System Use and Examples. Remote Sensing 8(11), 938.

Ward, M., Jones, R., Brender, J., de Kok, T., Weyer, P., Nolan, B., Villanueva, C. und S. van Breda (2018): Drinking Water Nitrate and Human Health: An Updated Review. International Journal of Environmental Research and Public Health 15(7), 1557.

Wick, K., Heumesser, C. und E. Schmid (2012): Groundwater nitrate contamination: Factors and indicators. Journal of Environmental Management 111(3), 178-186.

Withers, P., Neal, C., Jarvie, H. und D. Doody (2014): Agriculture and Eutrophication: Where Do We Go from Here? Sustainability 6(9), 5853-5875.

ZAMG (2017): Zentralanstalt für Meteorologie und Geophysik, Messdaten Station „Engelhartstetten“. 